Right ; Dexamethasone-treated : The $z$. fasciculata shows irregular cell cords and large droplets in cells.

Fig. 2 Distribution of lipid droplets (being stained with Sudan Black B) in fasciculatal cells of adrenal cortex under 1 week treatment.

Left ; control : The same finding as 5 days treatment.

Right : Dexamethasone-treated: The z.fasciculata shows fine droplets and partial disappearances.

Fig. 3 Distribution of alkaline phosphatase activity (Substrate; Naphthol AS-MX Phosphate) in fasciculatal endothels of adrenal cortex under 5 days treatment.

Left ; control : Note the endothelial lining cells of intense activity.

Right : Hydrocortisone-treated : This enzymatic activity is depressed in the endothelial lining cells of $z$. fasciculata.

Fig. 4 Distribution of G6P-dehydrogenase in fasciculatal cells of adrenal cortex under 2 weeks treatment.

Left ; control ; Note areas of intense local activity in the cells of the $z$. fasciculata. Activity is showed with fine and dense granules.

Right ; Dexamethasone-treated : Enzymatic activity is depressed in the 2 . fasciculata. The cells showed atrophy, irregular appearance and coarse granules.

Fig. 5 Distribution of TPN-diaphorase activity in fasciculatal cells of adrenal cortex under 2 weeks treatment.

Left ; control :The cells in $z$. fasciculata show intense activity, and have fine and coarse granules. The cells with weak activity is intermixed.

Right; Paramethasone-treated : The enzymatic activity is a little depressed. On the whole, those findings are as same as control.

\title{
Histochemistry of the Adrenal Gland-the First Report
}

\author{
Yoshio Aso, Yoshinobu Hoshino and Hisao TAKaYasu \\ From the Department of Urology, Faculty of Medicine, University of Tokyo, \\ Hongo, Tokyo, (Chief : Prof. Hisao Takayasu)
}

As a forestep to study the various diseases of the human adrenal gland, the adrenal glands of rat were investigated with several histochemical stainings after various treatments of the animals.

\section{Experiment and Staining Methods}

Twenty adult female albino rats weighing 190 to $250 \mathrm{gms}$. were used for this experiment. They were divided into the following four groups.

Group 1: six of them received no treatments and were considered as the control group. Bilateral total adrenalectomy was performed in three of them before the experiment started and in the rest of them after the end of the experiment.

Group 2: 2 units of ACTH were given intramuscularly in six rats every day for 5 consecutive days. Bilateral adrenal glands were excised under nem- 
butal anesthesia 24 hours after the last injection.

Group $3: 2.5 \mathrm{mg}$ of cortisone was intramuscularly injected daily for 7 consecutive days in six rats. They were bilaterally adrenalectomized 24 hours after the last injection.

Group 4: in two rats, $10 \mathrm{mg}$ of metopirone was injected intraperitoneally four times every two hours. Bilateral adrenalectomy was performed in them under general anesthesia with nembutal 1 hour after the last injection.

The adrenal glands were stained as follows;

(1) Hematoxylin-eosin (H-E)

(2) PAS

(3) Sudan-black

(4) Methylgreen-pyronin ${ }^{1-3)}$

(5) Chromaffine substances with $\mathrm{K}_{2} \mathrm{Cr}_{2} \mathrm{O}_{7}-\mathrm{K}_{2} \mathrm{CrO}_{4}^{1,2,4)}$

(6) Alkaline phosphatase ${ }^{1,2,5)}$

(7) Acid phosphatase ${ }^{1,2,5)}$

(8) Nonspecific esterase ${ }^{1,2,6)}$

(9) 3 beta-hydroxysteroid dehydrogenase ${ }^{1,2,7)}$

H-E staining was done after formalin fixation. PAS and Methylgreenpyronin stainings were carried out after Carnoy fixation. Sudan black, chromaffine substances and enzyme stainings were done with fresh sections. The enzymes were stained also after sections were embedded in soft paraffin following cold acetone fixation.

Tissue sections were cut with the microtome to the thickness of 6 to 10 micrones.

\section{Results}

This paper reports a few interesting findings in H-E, methylgreen-pyronin, alkaline phosphatase and 3 beta-hydroxysteroid dehydrogenase stainings among all the above stainings.

1. H-E staining: Fig. 1 shows the normal structure of the adrenal gland. In ACTH (Fig. 2) and metopirone (Fig. 3) groups, there were decrease of clear cells and increase of compact cells in the fasciculate zone. The glomerular zone did not change or was slightly thinned.

All the cells were atrophied in the corticoid group (Fig. 4).

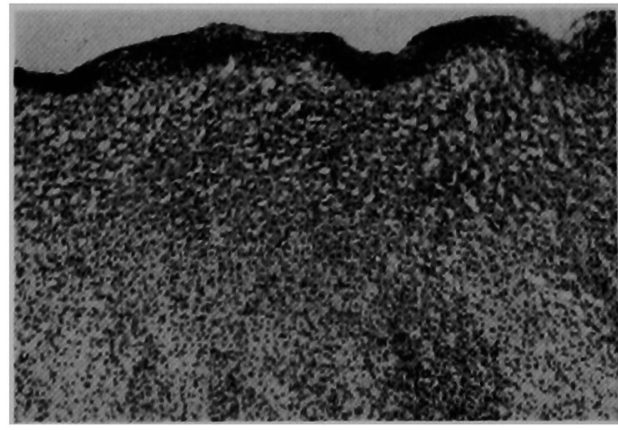

Fig. 1 H-E staining-normal.

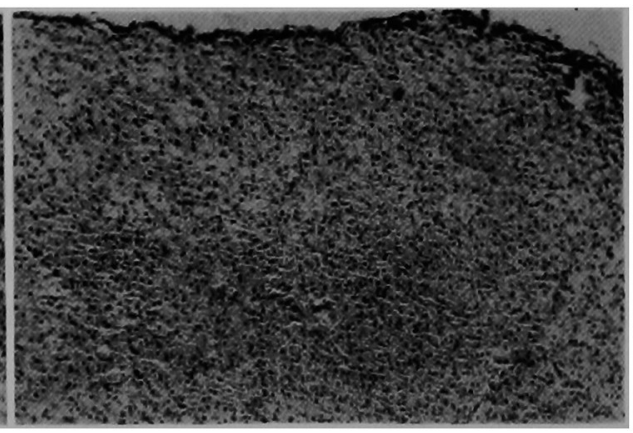

Fig. 2 H-E staining-ACTH group. 


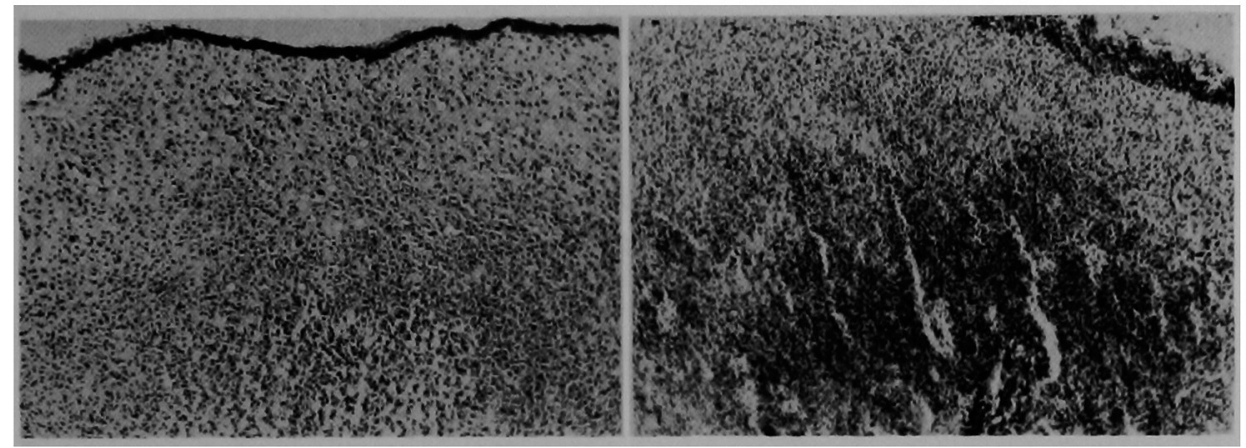

Fig. 3 H-E staining-metopirone group.

Fig. 4 H-E staining-corticoid group.

2. Methylgreen-pyronin : little changes were noted in ACTH group (Fig. 5). In corticoid group (Fig. 6), methylgreen staining appeared to be increased while pyronin staining decreased. Pyronin staining seemed to increase slightly in metopirone group (Fig. 7).

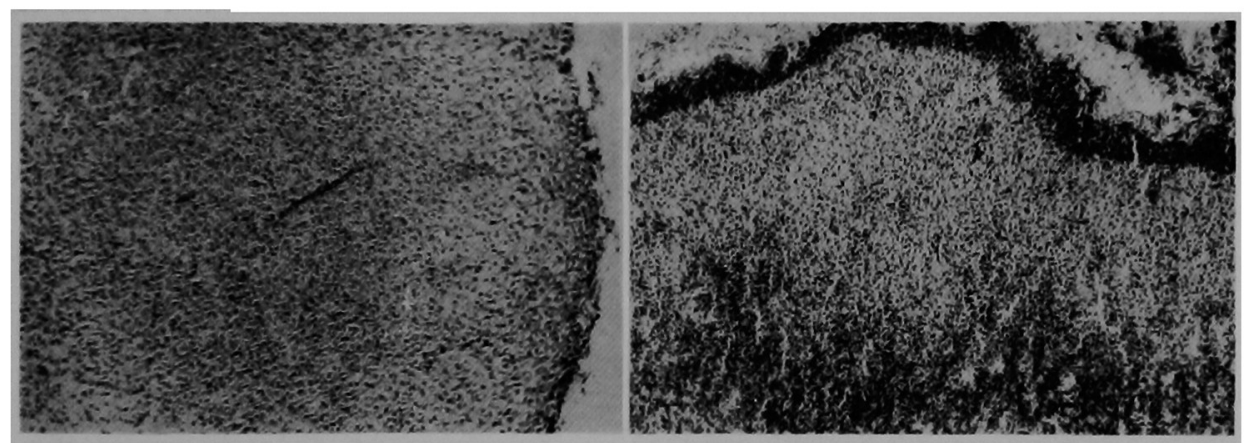

Fig. 5 Methylgreen-pyronin stainingACTH group.

Fig. 6 Methylgreen-pyronin stainingcorticoid group.

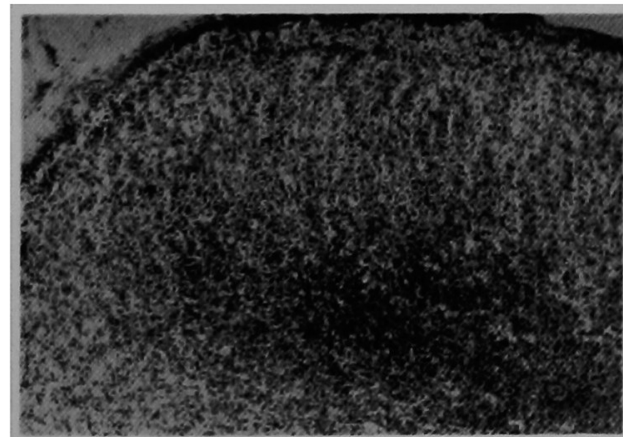

Fig. 7 Methylgreen-pyronin stainingmetopirone group.

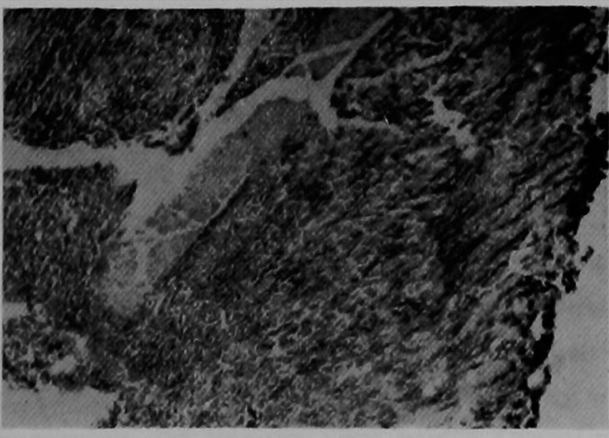

Fig. 8 Alkaline phosphatase-ACTH group.

3. Alkaline phosphatase: the control group showed the activity of this enzyme mainly in the glomerular zone. In the ACTH group (Fig. 8), the activity in the glomerular zone increased, while that in the other zones did not change or increased. The corticoid group (Fig. 9) showed the decreased ac- 
tivity in the other zones than in glomerulosa. The metopirone group revealed the same tendency as the group of ACTH administration.

4. beta-hydroxysteroid dehydrogenase: the activity of this enzyme was observed mainly in glomerular and fascicular zones (Fig. 10). No changes of this enzymatic activity and distribution were seen in ACTH (Fig. 11), cortisone (Fig. 12) and metopirone administrations.

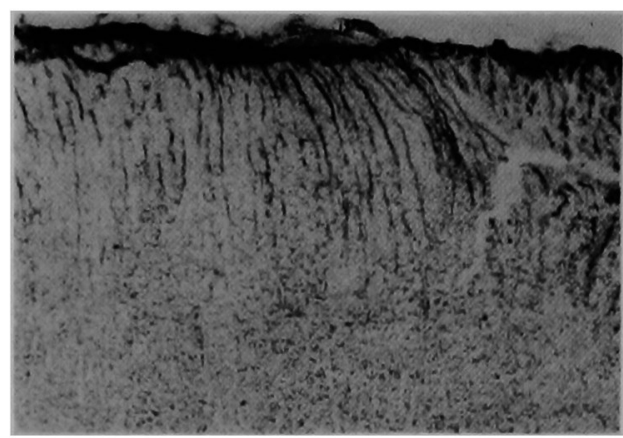

Fig. 9 Alkaline phosphatase-corticoid group.

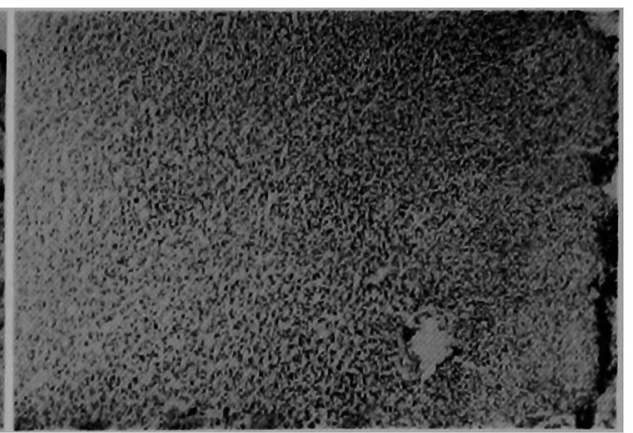

Fig. 103 beta-hydroxysteroid dehydrogenase-normal.

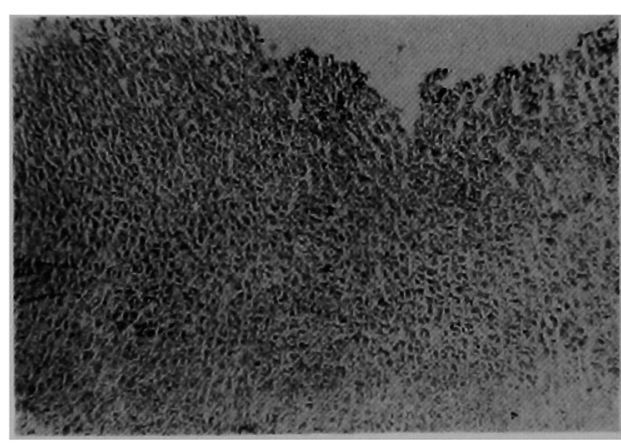

Fig. 113 beta-hydroxysteroid dehydrogenase-ACTH group.

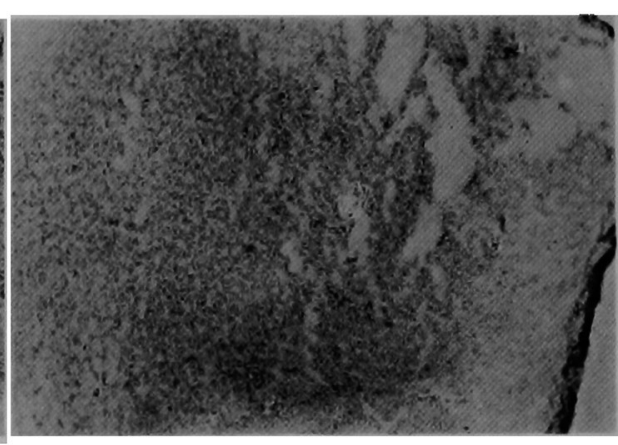

Fig. 123 beta-hydroxysteroid dehydrogenase-corticoid group

\section{Discussion}

It is not known what part of adrenal functions the substances stained with methylgreen-pyronin and alkaline phosphatase play roll in. An interesting finding is that 3 beta-hydroxysteroid dehydrogenase showed no changes by the various treatments. In other words, the activity of the enzyme relating with steroids does not change even when hyper- or hypo-function of the adrenal gland was caused by treatments. Those are substances like lipoids, cholesterol and other enzymes that show changes by the treatments. 3 betahydroxysteroid dehydrogenase is accumulated in the gland with some reserve so as to adapt to both hyper and hypo-functions of adrenal glands. This may be called "enzyme reserve" in the adrenal gland.

In hypo-functional adrenal gland, methylgreen staining increased as compared with pyronin staining. The functional status might be determined to 
some extent by this staining. However, this decrease of methylgreen staining would be seen only to some degree of hypo-function. If atrophy of the gland is far advanced, methylgreen staining would also diminish or disappear.

Further investigation is necessary to know the relationship between adrenal function and the production of nucleic acids.

\title{
Conclusion
}

(1) Some stainings seem to be valuable to know adrenal function.

(2) 3 beta-hydroxysteroid dehydrogenase is accumulated in the gland with some reserve to adapt to the functional change of the gland-"enzyme reserve" in the adrenal gland.

(3) So many points concerning adrenal histochemistry and function are still left to be solved in the future.

\section{Bibliography}

1) Pearse, A. G. E. : Histochemistry-Theoretical and Applied. Little, Brown and CompanyBoston, 1961. 2) Lison, L. : Histochemie et Cytochemie Animales, Principe et Méthodes, 3e édition, Guthier-Villars. \& Co., Paris, $1960 . \quad 3)$ Kurnick, N. B. : Int. Rev. Cytol., 4, 221, $1955 . \quad 4$ ) Hillarp, N. A. \& Hökfelt, B. : J. Histochem. Cytochem., 3, 1, $1955 . \quad 5)$ Manheimer, L. H. and Seligman, A. M. : J. Nat. Cancer Inst., 9, 181, $1949 . \quad$ 6) Shnitka, T. K. and Seligman, A. M.: J. Histochem, Cytochem., 9, 504, 1961. 7) Fuhrmann, K.: Arch. Gynåk., 197, 583, 1963.

\section{Electron Microscopic Studies of Kidney with Periodic Acid Silver Methenamine Staining.}

\author{
Kenzo Oshima, Michinobu Hatano, Yutaka Oikawa \\ and Toru Akamine
}

Oshima Clinic of Medicine, Nihon University, School of Medicine, Tokyo

The metal staining of ultrathin sections, such as phospotungstic acid, lead and uranyl acetate stainings, are well known for increasing the electron density of fine cellular components, and recently silver staining method has also developed.

$\mathrm{OsO}_{4}$ conjugated with unsaturated fatty acids in each membranous component of cells is assumed to reduce ammoniacal silver and results in heavy staining of protoplasmic membranes. And the pretreatment of the thin sections with periodic acid releases the conjugation of $\mathrm{OsO}_{4}$ with fatty acids and decreases the staining activity of protoplasmic components and, on the other hand, metaplasma such as basement membrane is heavily stained. 